\title{
Optimization of Single-Satellite Operational Schedules Towards Enhanced Communication Capacity
}

\author{
Sara Spangelo * and James Cutler ${ }^{\dagger}$
}

\begin{abstract}
There are a growing number of small satellites that are being launched and operated to accomplish novel science and technology missions. Scientists and mission operators are seeking to download large quantities of data from these small satellites, however they are resource-constrained and communicate to capacity-constrained ground networks. Motivated by the need for intelligent operational schedules for these missions, in this paper we develop an optimization formulation with the objective to maximize the data returns of a single satellite communicating to a global ground station network. We include decisions on when and how to perform satellite operations, including collecting missionspecific data by the payload and downloading data to ground stations. The formulation captures dynamic satellite states, subsystem functions, and interactions of the satellite with the external environment and ground station networks. The formulation is generic and extensible such that it is applicable to diverse mission scenarios. We apply the formulation to optimize the operations of realistic small satellite missions communicating to diverse ground networks. We demonstrate the advantages of optimizing operational schedules relative to using a conventional requirements-driven approach for operational planning. We use this formulation to perform an initial design space exploration relative to both deterministic and stochastic mission parameters.
\end{abstract}

\section{Introduction}

The potential for innovative small space mission architectures to accomplish novel science and technology missions is being realized worldwide [1], and in response, there is a growing community of small satellites being designed, built, and launched [2, 3, 4]. For example in the United States, the National Science Foundation (NSF) has sponsored multiple nanosatellite missions to explore space weather phenomenon [5, 6, 7]. In addition, NASA is developing and launching a variety of satellites at their research centers and also providing launches to over thirty nanosatellites (satellites that are 1-10 kg) through their Educational Launch of Nanosatellites (ELaNa) program [8]. There is also an emerging trend towards proposing and launching constellations of globally distributed small satellites to perform distributed, in-situ remote sensing, for example the ARMADA, High-Latitude Dynamic E-Field (HiDEF) Explorer, and QB50 projects all propose to launch dozens of small satellites to accomplish novel science objectives [9, 10].

These single and constellation small satellite missions are collecting large quantities of valuable science data, which scientists and satellite operators seek to download. However, the ability of these satellites to perform payload operations, process data, determine and control their position and attitude, recover from failures, and download payload and telemetry data to ground stations are restricted by their limited mass, size, volume, power, and funding levels [11]. In addition, the existing ground communication infrastructure for these missions is often unreliable and inefficient. Most small satellite missions have only a single or handful of low-cost and low-gain stations, which may only be available or functional a fraction of the time [12]. The growing community of highly-constrained small satellite missions seeking to downlink greater amounts of data coupled with the limitations of ground station infrastructure leads to important operational and scheduling challenges. In response to these challenges, in this paper we formulate and solve the Single-Satellite Operational Scheduling Problem (SOSP), where the objective is to schedule a satellite towards maximizing data download, taking into account realistic mission dynamics and constraints.

Next we provide a brief review of the literature in the area of satellite operational scheduling in the context of the contributions of this paper. There is a great deal of literature in the area of the Earth Observing Satellites (EOS) scheduling problem. In $E O S$, the goal is to take the maximum number of high-priority observations with fixed onboard spacecraft sensors during a given time interval. The EOS problem is similar to SOSP in that they both consist of scheduling a set of complex tasks involving the exchange of limited resources between an orbiting spacecraft and Earth-based targets. Additionally, data and energy are collected and consumed in both problems, constraining when

\footnotetext{
${ }^{*}$ Ph.D. Candidate, Aerospace Engineering, University of Michigan, 1320 Beal Ave, Ann Arbor, MI 48109

${ }^{\dagger}$ Assistant Professor, Aerospace Engineering, University of Michigan, 1320 Beal Ave, Ann Arbor, MI 48109.
} 
and how tasks can be performed. The EOS problem is often generalized and formulated with a common problem structure, such as a knapsack problem [13, 14], a packing problem [15], a single-machine scheduling problem [16], or a network flow problem [17]. Other common approaches to solve the EOS problem include constraint programming [14, 18], greedy algorithms based on spacecraft priorities [14, 15], dynamic programming [14, 17], heuristic approaches [19, 20, 21], and genetic algorithms [22, 15, 20]. Despite the similarities between EOS problem and the SOSP, there are noteworthy differences in the problem objectives, decisions, and constraints. SOSP must take into account the coupling between the decisions to perform payload operations and download. Furthermore, in SOSP, we often have to decide between multiple options when performing a function, for example which data rate to use or ground station to communicate with. Thus the satellite operational scheduling must be modeled and solved in a new way, however much of the EOS and download scheduling literature is informative in developing these models and algorithms.

Only a handful of researchers have studied the problem of scheduling spacecraft downlinks to Earth-based stations, however spacecraft energy collection, storage, and consumption are neglected in this work [20, 23, 21]. In fact, much of the theoretical literature towards designing, optimizing, and managing satellite schedules uses simplified models and fails to include logical constraints, on-board data storage, communication systems, and energy management systems $[14,17,15]$. Many models for scheduling optimization of satellite operations, such as the EOS problem, assume there are no precedence or logistical constraints $[15,19]$. Existing modeling approaches often neglect key elements required for end-to-end space system modeling. These simplifying assumptions reduce model fidelity and fail to capture complex interactions between the subsystems and with the external environment. Thus, their applicability to real systems is severely limited, and in sufficient for deployment to our existing satelite systems.

In response to the limitations of existing models and optimization formulations for scheduling satellite operations in the literature, we have developed an analytical and extensible modeling framework and simulation environment in Ref. [24]. Our modeling framework enables analytic assessment of communication capacity and optimization of operational plannin with applicability to diverse and realistic missions and ground networks. Based this framework, we introduced an initial formulation for the satellite communication problem, the Single-Satellite Multiple-Ground Station Scheduling Problem (SMSP) in Ref. [25]. This formulation only includes download decisions, neglecting other operational decisions that may constrain the ability of the spacecraft to download. Thus, a generalization of this formulation is necessary to capture a broader set of satellite operational decisions, subsystems, and states.

In this current paper, we formulate and solve the Single-Satellite Operational Scheduling Optimization Problem (SOSP). SOSP is a generalization and extension of SMSP based on our foundational modeling framework [25, 24]. $S O S P$ provides a general template for scheduling any type and number of operational decisions, thus capturing the coupling between all operational decisions, for example those related to payload and download. The objective is to maximize data downloaded and realistic space mission, and captures logistical and storage constraints neglected in much of the literature. We apply the scheduling formulation to optimize diverse mission architectures and demonstrate the advantages of including the operational decisions for intelligent use of highly-constrained resources on-board small satellites. Next, we exploit the ease of solving SOSPs with linear programming techniques to perform a preliminary design space exploration for representative missions. We investigate the sensitivity of the download performance relative to deterministic input parameters such as power generation, battery capacity, ground station network size, and data rate. Next, motivated by the uncertainty of operating this class of mission, we investigate the robustness of download performance relative to stochastic data download efficiencies. For this stochastic analysis, we use historic download efficiency data from a representative small satellite mission and ground networks using various strategies for modeling download efficiency.

\section{Optimization Problem}

\section{A. Problem Description}

The objective of SOSP is to maximize the amount of data collected from a single satellite and downloaded to a network of geographically and functionally diverse ground stations. The decisions include when and how to perform mission operations. Our formulation provides templates for any operational decisions. In our implementation of the formulation we focus on two types of decisions: payload functions, which generally consists of acquiring data, and download functions, which generally involves communicating data to ground stations. Opportunities for payload and download functions and exist according to spatial or temporal constraints. As the satellite orbits the Earth, targets of interest and ground stations come in and out of view, providing these opportunities, see Fig. 1a for a schematic. Opportunities for solar power collection depend on when the satellite is in the sun or in eclipse. These opportunities can be estimated a priori since we have good knowledge of the satellites' orbital position.

Functionally, the satellite consists of the subsystems that keep it operating nominally and one or multiple payloads, 
which accomplish the scientific, observational, or engineering mission goals. Satellite states include position, attitude, on-board stored energy, data, temperatures, and their time-dependent derivatives. The subsystems have functions that operate on the satellite states. An example function is downloading on-board data to a ground station. Each function has a set of available functional options. The set of available transmit data rates are example download options.

Satellite commodities, $c \in C$, are satellite states necessary to support the problem objectives and constraints. Example commodities include the amount of on-board stored energy and data and the satellite temperature states. Commodities are collected from the environment, exchanged by the subsystems towards satisfying mission requirements and achieving mission objectives, and may be able to be stored on-board in finite buffers for future use.

There are two challenging characteristics of the satellite scheduling problem. First, the realistic satellite communication problem is continuous in time because the orbit and attitude dynamics, and thus the energy and data acquisition and consumption, are continuous. Second, there is a discontinuity in the problem dynamics caused by the upper bound on the allowable amount of stored commodities, which results in a difficult non-linear optimization problem.

To overcome these challenges, we discretize the problem into a finite set of intervals $I$ that span the planning horizon, duration of time we want to schedule, to approximate the continuous-time dynamics. By definition, within an interval the set of functions and options for performing those functions remains constant. For example, the ground stations in view of the satellite remain constant during an interval. Whenever a target of interest or ground station comes in or out of view of the spacecraft, or the satellite goes into or out of eclipse (we assume this occurs at a discrete point in time), a new interval starts. Every interval $i \in I$ has a start time, $t_{i}$, and an end time, $t_{i+1}$. The end of one interval coincides with the start of the next interval and the duration of every interval is $\Delta t_{i}=t_{i+1}-t_{i}$. Intervals with at least one ground station in view of the spacecraft are download opportunities and those with a target of interest in view are data collection opportunities. See Figure 1a for an example showing the ground stations in view of the satellite and $1 \mathrm{~b}$ showing an example of how the intervals are defined.

The challenge of a discontinuous upper capacity constraint is managed by imposing a commodity storage constraint at the start and end of each interval. This is an Under-Constrained (UC) approach to the formulation, based on the $S M S P$ formulation developed in Ref. [25]. The formulation does not prevent constraint violations that may occur at any intermediate times throughout the interval. We have shown that when the commodity dynamics are linear throughout an interval, such as when energy and data are collected at a constant rate, the UC formulation guarantees optimality, and thus feasibility, of the solution to SMSPS [25]. This result is applicable to the SOSP formulation because of the underlying similarity in the problem structure and linearity of the dynamics.

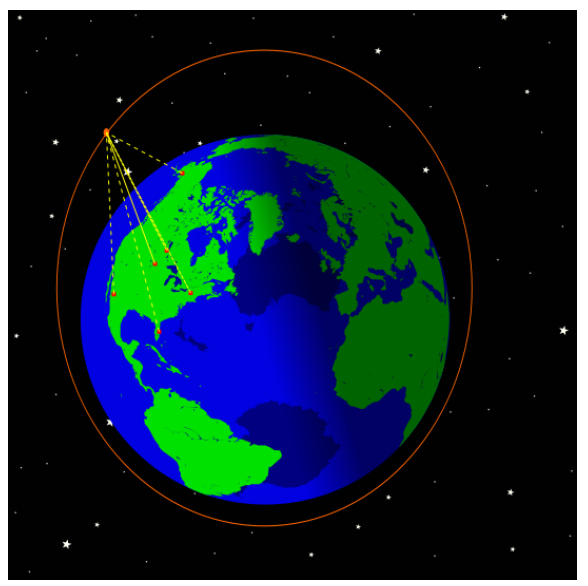

a) Opportunities for downloading to ground stations and collecting data from targets of interest.

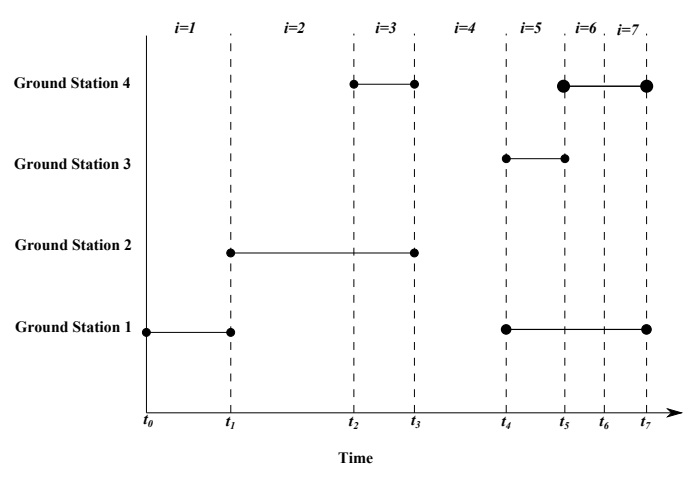

b) Intervals ( $i$ ) and download opportunities to ground stations.

Figure 1. Schematics describing the Single-Satellite Operational Scheduling Optimization Problem (SOSP).

\section{B. Problem Formulation}

We introduce and define necessary problem sets, parameters, variables used in the analytic problem formulation. Sets and Subsets

- $I$ is the set of intervals over the planning horizon

- $F$ is the set of all satellite functions that have operational decisions

- $F_{i} \subseteq F$ is the subset of functions with opportunities to operate during interval $i, \forall i \in I$

- $C$ is the set of all commodities 
- $C_{f} \subseteq C$ is the subset of commodities operated on by function $f, \forall f \in F$

- $O_{f}$ is the set of download options for function $f, \forall f \in F$

- $O_{i f} \subseteq O$ is the subset of download options available during interval $i$ using function $f, \forall i \in I, \forall f \in F$

\section{Parameters}

- $t_{i}$ is the start time of interval $i$, measured in seconds, $\forall i \in I$

- $t_{i+1}$ is the end time of interval $i$, which coincides with the start of interval $i+1$, measured in seconds, $\forall i \in I$

- $\Delta t_{i}$ is the duration of time interval $i\left(\Delta t_{i}=t_{i+1}-t_{i}\right)$, measured in seconds, $\forall i \in I$

- $z_{c, \min }$ and $z_{c, \max }$ are the minimum and maximum storage capacity of commodity $c$, measured in units of commodity $c$, $\forall c \in C$

- $z_{c, \text { start }}$ is the amount of commodity $c$ stored on-board the satellite at the beginning of the planning horizon, measured in units of that commodity, $\forall c \in C$

- $\delta_{i c}^{+} \geq 0$ is the amount of commodity $c$ acquired nominally (independent of operational decisions) during interval $i$, measured in units of that commodity, $\forall i \in I, c \in C$

- $\delta_{i c}^{-} \geq 0$ is the amount of commodity $c$ consumed nominally (independent of operational decisions) during interval $i$, measured in units of that commodity, $\forall i \in I, c \in C$

- $G_{i f c o}$ is the maximum allowable transfer of commodity $c$ due to function $f$ using option $o$ during interval $i$ in units of that commodity, $\forall i \in I, f \in F_{i}, c \in C_{f}, o \in O_{i f}$.

- $\phi_{i f c o}$ is the linear rate of change of commodity $c$ due to function $f$ using option $o$ during interval $i$ in units of that commodity per second $\forall i \in I, f \in F_{i}, c \in C_{f}, o \in O_{i f}{ }^{1}$

\section{Variables}

- $x_{i f o} \in\{0,1\}$ is the binary variable representing the decision to perform function $f$ using option $o$ during interval $i$ (unity represents the decision to perform the operation while zero represents the decision not to), and it is unitless, $\forall i \in I, f \in$ $F_{i}, o \in O_{i f}$

- $q_{i f c o} \in \mathbb{R}$ is the amount of commodity $c$ transferred (acquired or consumed) by function $f$ using option $o$ during interval $i$, in units of that commodity, $\forall i \in I, f \in F_{i}, c \in C_{f}, o \in O_{i f}$

- $z_{i c} \in \mathbb{R}^{+}$is the amount of commodity $c$ stored on-board the satellite at the beginning of interval $i$, measured in units of that commodity, $\forall i \in I, c \in C$

- $h_{i c} \in \mathbb{R}^{+}$is the amount of excess commodity $c$ spilled during the interval $i$, measured in units of that commodity, $\forall i \in$ $I, c \in C$

The objective of the optimization problem is to maximize the amount of data downloaded, identified by commodity $c^{*}$, from the satellite to the ground station network over the planning horizon, expressed mathematically as the sum of the download overall the intervals, $i \in I$ in Eq. 1. The optimization problem is subject to Constraints 2-8.

$$
\max z_{n c^{*}}
$$

s.t.

$$
\begin{aligned}
\sum_{o \in O_{i}} x_{i f o} & \leq 1 \\
z_{c, 0} & =z_{c, s t a r t} \\
z_{c, \text { min }} & \leq z_{i c} \leq z_{c, \max } \\
z_{i+1, c} & =z_{i c}+\delta_{i c}^{+}-\delta_{i c}^{-}+\sum_{f \in F_{i}} \sum_{o \in O_{i f}} q_{i f c o}-h_{i c} \\
q_{i f c o} & \leq G_{i f c o}\left(x_{i f o}, \Delta t_{i}\right) \\
x_{i f o} & \in\{0,1\} \\
q_{i f c o} & \in \mathbb{R}^{+}
\end{aligned}
$$

$$
\begin{array}{r}
\forall i \in I, f \in F_{i}, o \in O_{i f} \\
\forall c \in C \\
\forall i \in I, c \in C \\
\forall i \in I, c \in C \\
\forall i \in I, f \in F_{i}, o \in O_{i f}, c \in C \\
\forall i \in I, f \in F_{i}, o \in O_{i f} \\
\forall i \in I, f \in F_{i}, o \in O_{i f}, c \in C
\end{array}
$$

Constraint 2 enforces that during every interval $i$, each function $f$ can only operate using a single option $o$ from the set of options available for that function during that interval, $O_{i f}$. Constraints 3-5 enforce the storage and dynamics for

\footnotetext{
${ }^{1}$ This parameter only exists if the rate of change of the commodity $c$ is constant.
} 
the set of commodities, $C$, for each interval $i \in I$. Constraint 3 initializes the quantity of stored commodity $c$ stored onboard the satellite at the start of the planning horizon $(i=0)$ and Constraint 4 enforces that the amount of commodity $c$ never violates the upper and lower bounds storage constraints. Constraint 5 updates the amount of stored commodity $c$ on-board the satellite at every interval $i$ based on nominal dynamics and the executed functions. The amount of commodity $c$ stored on-board the satellite at interval $z_{i+1, c}$ is equal to the amount stored at the previous interval, $z_{i c}$, plus any commodity $c$ acquired and consumed throughout the interval due to nominal dynamics (independent of subsystem functions), $\delta_{i c}^{+}-\delta_{i c}^{-}$, plus any change in the commodity $c$ due to subsystem functions (which may be positive or negative), minus any spillage of the commodity. Spillage of a commodity occurs to prevent overfilling a storage buffer,i.e. to prevent violating Constraint 4. Constraint 6 enforces that the amount of commodity $c$ transferred during interval $i$ by function $f$ and option $o$ is less than the maximum allowable transfer based on these conditions, $G_{i f c o}\left(x_{i f o}, \Delta t_{i}\right)$. In the case that commodity $c$ is transferred at a constant rate, Constraint 6 is linear and is expressed:

$$
G_{i f c o}\left(x_{i f o}, \Delta t_{i}\right)=\phi_{i f c o} \cdot x_{i f o} \cdot \Delta t_{i} \quad \forall i \in I, f \in F_{i}, c \in C_{i f}, o \in O_{i f},
$$

When Constraint 6 is linear and Eq. 9 is true, the complete optimization formulation in Eq. 1-8 is linear.

In this paper, we consider two optimization problems using the foundational formulation in Eqs. 1-8 to compare the existing SMSP formulation from the literature with the generalization of the formulation presented in this paper.

1. Optimal Download with Download Decisions (DL): In this formulation, the decision variables are only related to data download operations. This formulation is based on SMSP from Ref. [25].

2. Optimal Download with Operational Decisions (OPS): In this formulation, the decision variables are related to data downloads and payload operations such that the coupling of the operational decisions are considered.

\section{Implementing and Solving SOSP}

Implementation of the SOSP formulation requires the user to decide on an appropriate interval duration, which depends on the specific problem and available computational resources. Using very small intervals would enable the formulation to approach an accurate representation of the continuous-time dynamics, however it would result in the problem becoming computationally intractable, because as $\Delta t_{i}$ approaches zero, the number of intervals approaches infinity. Therefore, we consider $\Delta t_{i} \geq 1$ minute, $\forall i \in I$ in our examples as this is typically the smallest interval of time over which operational decisions related to the payload or communication system are practical.

For most satellite operational problems, it is reasonable to assume the dynamics are linear because the commodities we model (e.g. energy and data) are generally collected and consumed at constant rates throughout the 1 minute interval duration described above. Thus, we can exploit the problem structure and use linear programming techniques, as discussed in Section I.A. Linear programming involves solving the problem of minimizing a linear cost function subject to linear equality and inequality constraints. This approach is advantageous because it guarantees finding an optimal, and therefore feasible, solution if one exists for a given problem. Another advantage of using a linear formulation is that there are many commercially available optimization solvers which can be used to solve the problem in a computationally tractable and theoretically satisfactory way, such as CPLEX [26].

Our computations are performed on an Intel Core i $2.8 \mathrm{GHz}$ processor with $8 \mathrm{~GB}$ of memory using the IBM ILOG Optimization Studio (CPLEX) 12.1 Matlab API software package [26]. All test cases solve in under ten minutes. The computational issues of this problem is outside the scope of this paper, please see Ref. [25] for a more extensive discussion on the computational tractability of SMSP.

\section{Data Sets and Optimized Mission Results}

To demonstrate applicability of the formulation to realistic problems, Section III.A introduces the realistic satellite and ground station data sets and Section III.B shows optimization results for representative mission scenarios.

\section{A. Satellite and Ground Station Data Sets}

To obtain realistic data sets, we have deployed two surveys, one focusing on existing and upcoming small satellite missions and the other focusing on the ground stations supporting these satellites. These types of data sets are critical to verify our models and tools for realistic applications. Furthermore, optimizing realistic data sets provides insights about the performance potential and constraints of existing and future missions.

The Satellite Survey [27] is a database of operational information about past and future small satellite missions. Representative satellites and their operational parameters, extracted from the survey, are provided in Table 1. From 
our survey, we have identified three classes of data collection strategies. Type 1 uses a targeted data collection strategy, collecting data at known instances when in view of a target of interest. Type 2 uses a repeating data collection strategy, collecting data at known frequencies for known durations of time. Type 3 has continuous data collection. The Radio Aurora eXplorer (RAX) has Type 1 targeted data collection, collecting data when in view of groundbased radar stations, for example at Poker Flat, Alaska. The Naval Postgraduate School Solar Cell Array Tester (NPS-SCAT), Focused Investigations of Relativistic Electron Burst, Intensity, Range, and Dynamics (FIREBIRD), Michigan Multipurpose Minisatellite (MCubed), Kentucky Space's KySat, RApidprototypedMicroelectromechanicalsystem PropulsionAndRadiationTest CUBEflowSATellite (RAMPART) are Type 2 repeating data collection missions.

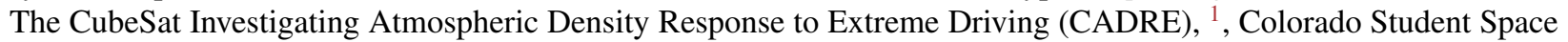
Weather Experiment (CSSWE), Dynamic Ionosphere Cubesat Experiment (DICE), Explorer - 1 [Prime] (E1P) are Type 3 continuous data collection missions. The satellites $n$ Table 1 are used in our optimization results in the coming sections.

The Ground Station Survey is a database of information on existing ground stations, including their locations and capabilities [28]. The ground station networks used to support small satellites are independently owned and operated and not centrally controlled. In our analysis we assume the satellites may only communicate to ground stations when the elevation angle from the horizon to the satellite location exceeds $30^{\circ}$, which is reasonable for most ground stations from our experience with the RAX-2 mission and efficiency data. We consider three classes of networks:

- True Ground Network (TGN): ground stations that are intended to support each mission, see Table 1.

- Large Ground Network (LGN): unique collection of 14 ground stations that are intended to support all the missions in Table 1.

- Very Large Ground Network (VLGN): collection of 30 globally distributed ground stations from the Ground Station Survey [28].

Table 1. Satellite mission parameters from survey [27]. The data collection strategies are: Type 1 is targeted, Type 2 is repeating, and Type 3 is continuous.

\begin{tabular}{|c|c|c|c|c|c|c|c|c|c|c|c|}
\hline Mission Parameter & Units & RAX-2 & CADRE & CSSWE & $\begin{array}{l}\text { NPS- } \\
\text { SCAT }\end{array}$ & DICE & $\begin{array}{l}\text { FIRE- } \\
\text { BIRD }\end{array}$ & MCubed & KySat & $\begin{array}{c}\text { RAMP- } \\
\text { ART }\end{array}$ & E1P \\
\hline Data Collection Type & - & 1 & 3 & 3 & 2 & 3 & 2 & 2 & 2 & 2 & 3 \\
\hline Perigee Altitude & $\mathrm{km}$ & 330 & 500 & 450 & 700 & 350 & 550 & 330 & 630 & 350 & 350 \\
\hline Apogee Altitude & $\mathrm{km}$ & 810 & 500 & 800 & 500 & 820 & 550 & 810 & 640 & 1200 & 800 \\
\hline Orbital Inclination & degrees & 101.8 & 81 & 55 & 75 & 102 & 60 & 102 & 98 & 72 & 101 \\
\hline Maximum Energy Capacity & kJoules & 115 & 80.4 & $6 \mathrm{~s} 6.2$ & 36.9 & 72.0 & 58.3 & 58.6 & 57.6 & $1.35 \cdot 10^{5}$ & $1.04 \cdot 10^{5}$ \\
\hline Maximum Data Capacity & Gbits & 16 & 16 & 16 & 2 & 64 & 16 & 17 & 16 & 0.032 & 0.016 \\
\hline Maximum Depth of Discharge & $\%$ & 20 & 30 & 85 & 10 & 29 & 6 & 20 & 3 & 10 & 4.5 \\
\hline Nominal Power Collection (sun) & Watts & 7 & 15 & 7 & 1.5 & 3 & 3 & 1.5 & 1.5 & 3 & 3 \\
\hline Nominal Power Consumption & Watts & 0.75 & 8.5 & 0.15 & 0.75 & 1.5 & 1.5 & 0.25 & 0.75 & 1.5 & 0.75 \\
\hline Experiment Power Consumption & Watts & 3.6 & 1.3 & 0.25 & 0.25 & 1.5 & 1.5 & 5 & 0.25 & 1.5 & 0.25 \\
\hline Nominal Data Collection & Mbits/sec & $2 \cdot 10^{-4}$ & 0.2 & $7 \cdot 10^{-6}$ & $3 \cdot 10^{-6}$ & 0.01 & $2 \cdot 10^{-4}$ & $3 \cdot 10^{-6}$ & $3 \cdot 10^{-5}$ & $8 \cdot 10^{-6}$ & $10^{-6}$ \\
\hline Payload Data Collection Rate & Mbits/sec & 4 & $2 \cdot 10^{-4}$ & $5 \cdot 10^{-5}$ & $3 \cdot 10^{-6}$ & 0.01 & 0.005 & 50 & $3 \cdot 10^{4}$ & $3 \cdot 10^{-5}$ & $6 \cdot 10^{-6}$ \\
\hline Download Power Consumption & Watts & 3 & 10 & 5 & 3 & 1.5 & 3 & 0.25 & 1.5 & 5 & 0.25 \\
\hline Download Data Rate & $\mathrm{kbits} / \mathrm{sec}$ & 9.6 & 250 & 9.6 & 9.6 & 1500 & 0.22 & 9.6 & 9.6 & 9.6 & 1.2 \\
\hline Minimum Required Download & MBytes/day & 1.0 & 30 & 0.27 & 0.036 & 0.29 & 0.039 & 0.84 & 0.14 & 0.0021 & 0.016 \\
\hline Number of Ground Stations & - & 7 & 1 & 1 & 1 & 2 & 1 & 1 & 1 & 3 & 1 \\
\hline
\end{tabular}

\section{B. Mission Requirements Compared to Optimal Solutions}

Figure 2 compares the minimum download requirements to the results for the optimized schedules for representative satellites missions communicating to two ground station networks from see Section III.A. Results for the $D L$ and $O P S$ formulations are compared. The CADRE, DICE, and FIREBIRD missions are infeasible when the $D L$ formulation is used (this is why they do not have the second bar representing the $D L$ solution). This indicates that these missions can not support performing payload mission operations at every opportunity and must make payload operational decisions considering the full mission and upcoming events. For example, by performing energy-consuming operations earlier in the mission, the satellite may not be able to survive eclipse, when there is no opportunity for solar power collection. The ability of the optimized schedules to satisfy minimum download requirements can be assessed by comparison to the first bar for each mission in Figure 2. The RAX-2, CADRE, CSSWE, KySat, RAMPART, and E1P missions exceed

\footnotetext{
${ }^{1}$ For the CADRE mission, we assume the science experiment is part of the nominal operations, and the operational decisions are related to the operation of the GPS subsystem.
} 
the minimum download requirement using both the $D L$ and $O P S$ formulations while the NPS-SCAT, FIREBIRD, and MCubed missions are not able to achieve the minimum download requirement using either formulation.

Many of the missions show a significant increase in download potential when communicating to the Very Large Ground Network (VLGN), relative to when communicating to their True Ground Networks (TGN), see Figures 2a-2b to compare the networks. The NPS-SCAT and MCubed missions do not satisfy minimum download requirements when communicating to their TGNs, however exceed their requirements when communicating to the VLGN.

This analysis is useful for satellite designers and operators to realize the download potential when communicating to diverse networks, and may motivate the need to modify the vehicle, ground network, or operational parameters. The fraction of time the payload and download opportunities that are utilized and the buffer constraints are active can be extracted from the optimized schedules, and can provide insight into what factors limit the download potential. Section IV provides more insight to how the feasibility and download potential of these missions varies with problem parameters.

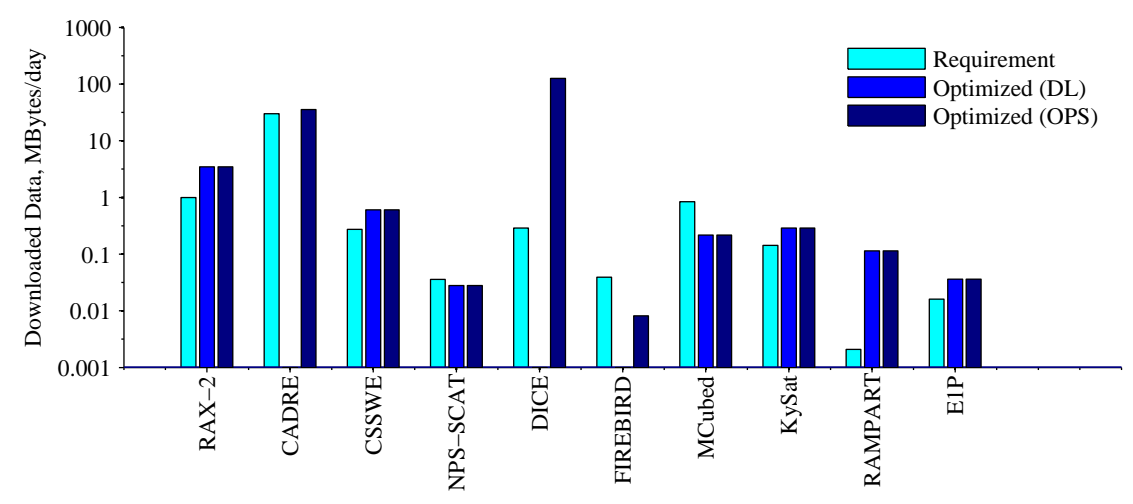

a) Results when satellites are communicating to their True Ground Networks with the number of ground stations from Table 1.

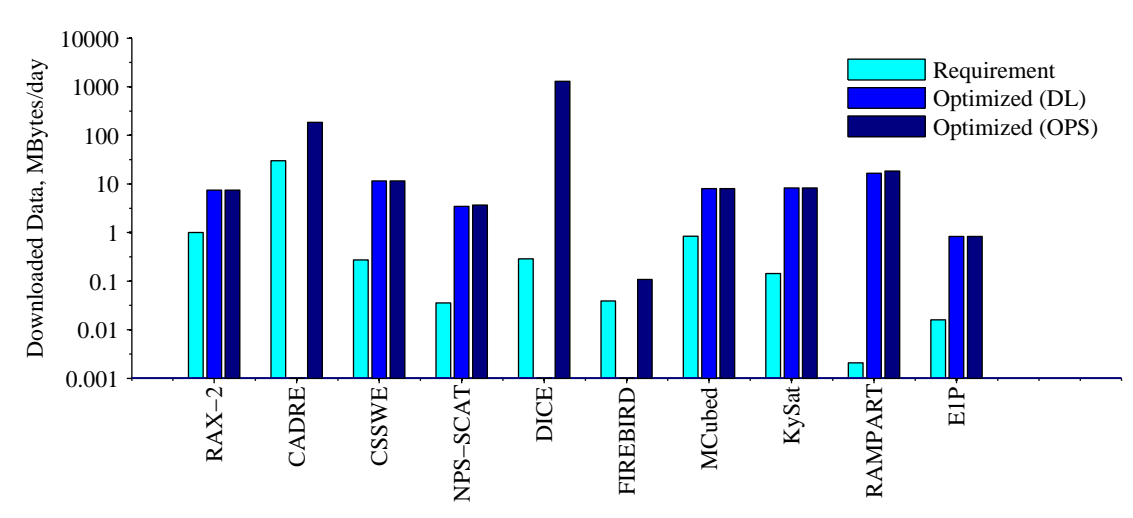

b) Results when satellites are communicating to the Very Large Ground Network consisting of 30 ground stations.

Figure 2. Comparison of mission download requirements to data downloaded with optimized schedules. Results are shown considering only the download decisions $(D L)$ and considering all operational decisions (OPS) for the satellite missions from Table 1 for a one day mission scenario assuming perfect download efficiencies $(\eta=1)$. Data downloads are shown on a log scale.

\section{Design Space Exploration}

Beyond enabling the optimization of satellite mission schedules, as seen in Section III, the optimization formulation from Section II also enables exploration of the satellite vehicle and operational design space. For small representative examples, SOSP solves very quickly using linear programming techniques (see Ref. [25]), enabling the rapid optimization and assessment of satellite scheduling problems with variable input parameters. This enables the satellite designers and operators to gain insight into the sensitivity of the solutions to input parameters. Sections IV.A and IV.B investigate the sensitivity of the optimized schedule downloads relative to deterministic and stochastic problem 
parameters, respectively. Using optimized schedules to perform design space trades in early design stages enables making informed and accurate spacecraft component sizing and logistical decisions. This is advantageous since design decisions can have great impact on later design and operational constraints and thus mission performance. In this section, the OPS formulation from Section II is used to solve for optimal solutions.

\section{A. Sensitivity to Deterministic Problem Parameters}

Next we investigate the sensitivity of optimized solutions for satellite schedules relative to deterministic vehicle and operational parameters. The parameters are deterministic because they have a known, constant, and quantifiable impact on the objective, which is the quantity of downloaded data. This is useful for identifying the relationship between mission parameters and the active constraints limiting the feasibility and download potential of the missions. Throughout this section we assume the download efficiency is perfect $(\eta=1)$. Sensitivity to stochastic download efficiency is investigated in Section IV.B.

\section{Power Generation and Battery Storage}

During spacecraft design, sizing the solar cell array and battery is challenging. This is because the energy state is dynamic and constrained by the coupling of the solar cell array and battery decisions, and difficult to model prior to making operational decisions. The optimization formulation presented in this paper enables the rapid optimization and analysis of how these two factors impact download performance, and can help make informed design decisions in the presence of minimum download requirements and constraints on spacecraft volume, mass, or cost.

Figure 3 shows the optimal download solutions for two representative missions from Table 1 with variable power generation and battery storage values. The combined effect of variation in battery depth of discharge (along the $\mathrm{x}$ axis) and collected power from the solar cells (along the y axis) demonstrates the coupling between these two parameters in constraining the energy available to support spacecraft operations. For example, higher power collection results in more available energy, but battery storage capacity may limit the amount of energy that can be stored for future use.

For both the CSSWE and DICE missions, the download performance is nearly independent of battery depth of discharge when the depth of discharge is sufficiently high, and for high values scales linearly with collected power. For lower depths of discharge there's a nonlinear trend in the download potential related to the trade-off between energy storage capacity and power collection. The same amount of data can be downloaded with less power and a greater depth of discharge as a case with higher power and a lower depth of discharge. For example, CSSWE mission in Figure 3a, see the curved line labelled 5.5 (representing data downloaded in MBytes/day) that curves dramatically for low battery depths of discharge.

The other satellite missions from Table 1 were not as sensitive to variability in power generation and battery storage. In most cases, once sufficient power is collected and there is a sufficient battery capacity, the solution was feasible, the same quantity of downloaded data was achieved, independent of these parameters.

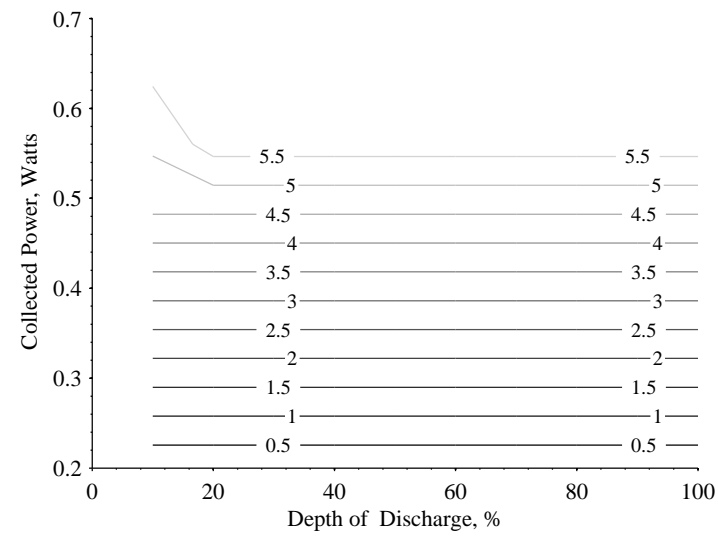

a) CSSWE

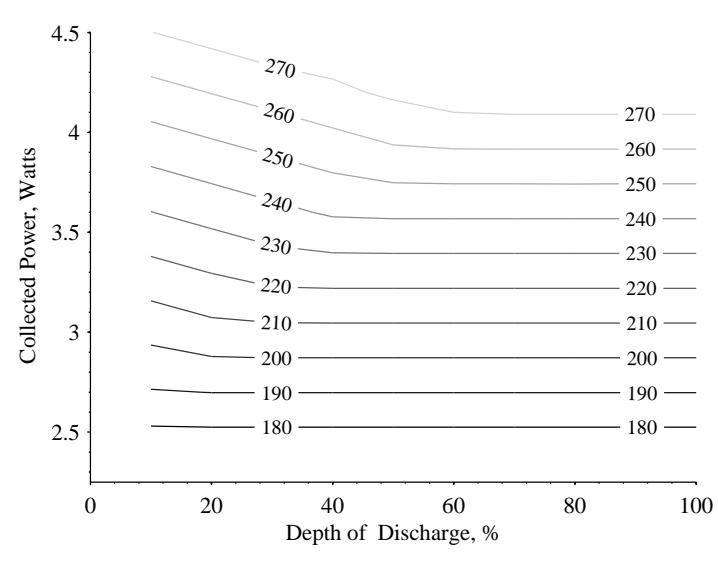

b) DICE

Figure 3. The sensitivity of optimized schedules relative to variations in power generation and battery depth of discharge. The lines on the contour plot represent the amount of data downloaded per day in MBytes/day. 


\section{Download Data Rate}

Most of the surveyed small satellite missions use relatively low- and constant-rate communication. This is due to the lack of existing higher- and variable-rate radios that are flight-ready or have flight heritage, and the lack of ground station infrastructure to support higher rate communication. However, emerging radio technology and ground station capabilities are enabling the future generation of spacecraft to use higher and variable-rate radios, thus we investigate the impact of variable data rates.

Figure 4 shows how the download potential scales with data rate for representative missions from the small satellite community from Table 1. We assume the power to download scales proportionally to changes in the download rate, which is a reasonable approximation for small satellite radios. Results are shown for each satellite mission communicating to its true ground network (TGN), from Table 1, and to the large ground network (LGN) described in Section III.A. These results help to identify if the nominal data rate (dotted vertical line) achieves the required download (solid horizontal line), and how variations in data rate impact download potential. The advantages of increasing the data rate can be extracted from these figures. For example, the data downloaded for the RAX-2 mission scales linearly with data rate for data rates less than $1 \mathrm{Mbit} / \mathrm{sec}$. When the data downloaded versus data rate curve plateaus, there are reducing returns for increasing the data rate, as seen for the RAX-2, CSSWE, and FIREBIRD missions. This occurs since these missions encounter an active constraint, such as limited availability to ground stations or energy limitations, which prevent an increase in download with data rate.

\section{Number of Ground Stations}

Most of the recently launched and upcoming small spacecraft missions rely on communicating to only a single or handful of ground stations, often at the university or institution where the satellite was built. However, there are existing and emerging ground station networks that have the potential to support multiple satellite missions, such as the loosely connected network of amateur ground stations [29], and more organized networks such as GENSO [30]. Thus we investigate the impact of growing ground station networks on download potential for representative missions. In this analysis, the 14 ground station network (LGN) described in Section III.A is used. The network grows by adding stations in a random order to represent realistic networks which grow by random addition of independently owned and operated ground stations. The download potential is computed for each network size.

The relationship between number of ground stations and quantity of downloaded data for representative missions is shown for both low $(<10 \mathrm{kbits} / \mathrm{sec})$ and high $(>100 \mathrm{kbits} / \mathrm{sec})$ data rate missions in Figures $5 \mathrm{a}$ and $5 \mathrm{~b}$, respectively. Although the download potential grows monotonically with additional stations, the trend is not continuous due to the random location of additional stations and availability of satellite commodities (such as energy and data) to support download. Future work will investigate how ground station location and other spacecraft constraints impact these results. The impact of larger networks on download potential is significant for satellites that are not energy-constrained, such as RAX-2, CSSWE, KySat, CADRE, and DICE. However, the impact of larger networks is not as significant for satellites that are constrained by available energy or data, which have similar download potential with a single stations as with a larger network, such as NPS-SCAT, FIREBIRD, and RAMPART.

\section{B. Sensitivity to Stochastic Download Efficiencies}

There are several sources of stochasticity in operating university-class small satellites missions due to the inherent uncertainties of low-cost, high-risk space vehicles supported by a low-cost ground network infrastructure in the challenging space environment. One of the greatest source of stochasticity for small satellite missions is download efficiency when communicating to ground stations. There are several factors that contribute to stochastic download efficiency, including unknown availability of ground stations because they are independently owned and operated, non-ideal antenna gain and orientation of the satellite relative to ground stations, hardware/ software ground station problems, and weather and external noise effects.

Statistical data based on historic data from the RAX-2 satellite communicating to seven globally distributed ground stations over approximately a one month time horizon in Figure 6. Download efficiencies are computed for each satellite pass, the time where the satellite is continuously above the horizon. Some stations have a large fraction of passes with high efficiencies, such as Adelaide, Australia and Wellington, New Zealand, while stations such as Fairbanks, Alaska are more likely to have low efficiencies than high efficiencies. Stations such as Sanford, North Carolina and Tokyo, Japan, are simply not available for large portions of the passes. ${ }^{1}$ Some of these ground stations have relatively

\footnotetext{
${ }^{1}$ These download efficiency values are preliminary. The efficiency values are very complex and a function of multiple factors, including the ground station availability, which is difficult to predict because the stations are independently owned and operated. We are currently working to improve the characterization of ground station efficiencies.
} 


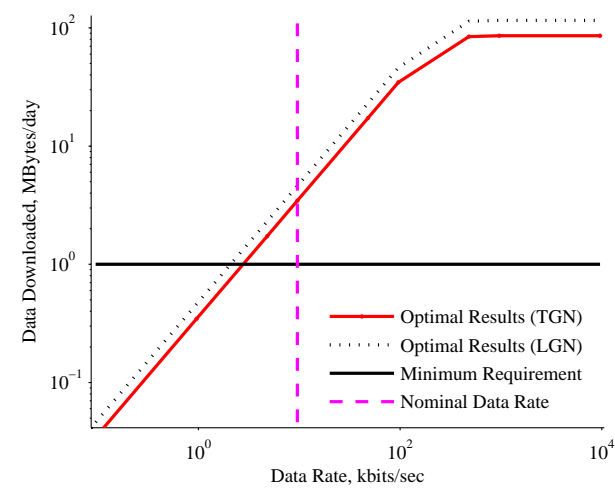

a) RAX-2

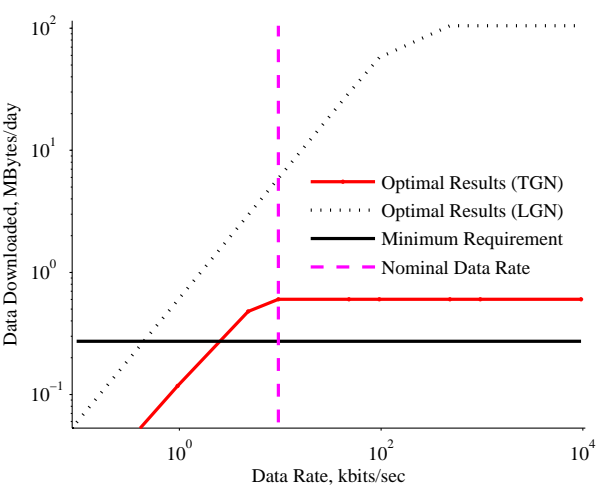

c) CSSWE

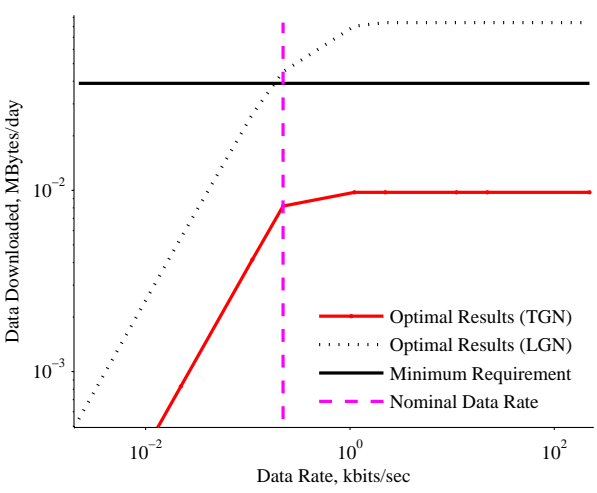

e) FIREBIRD

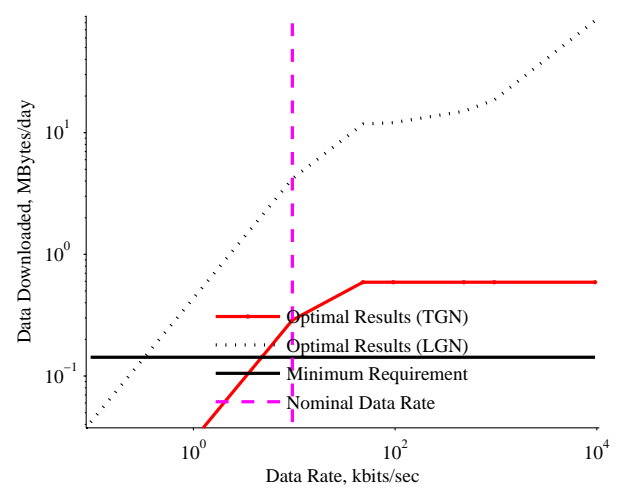

g) KySat

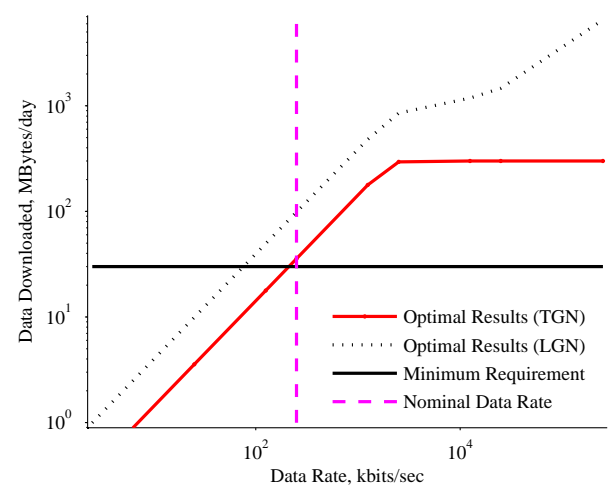

b) CADRE

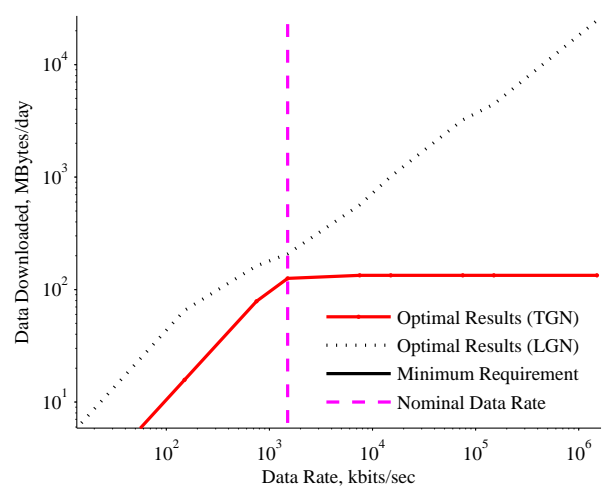

d) DICE

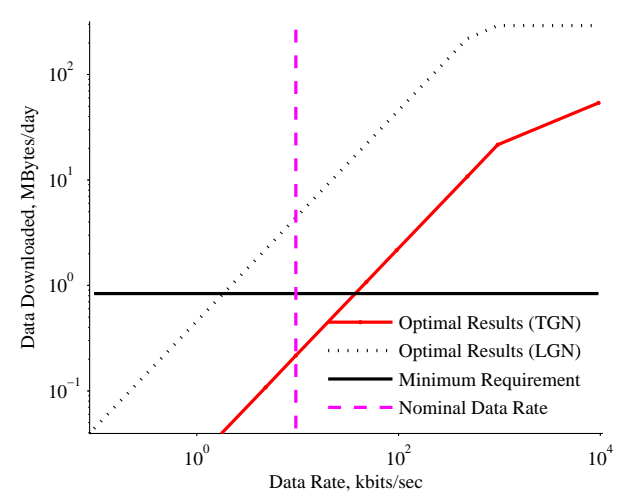

f) MCubed

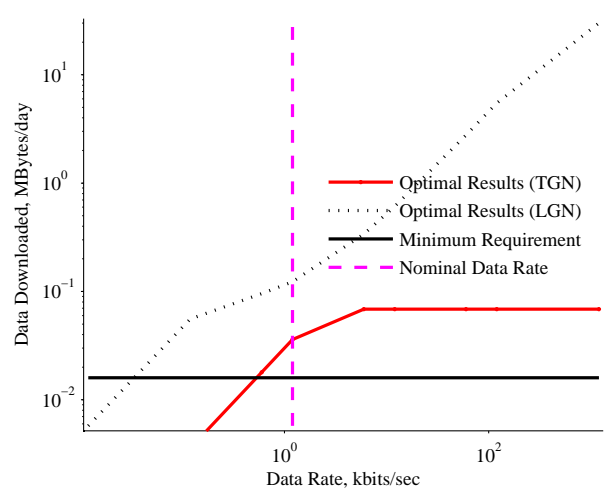

h) E1P

Figure 4. Sensitivity of data downloaded from optimized schedules to variations in download data rates. Optimal results are shown for each satellite communicating to its true ground network (TGN) and the large ground network (LGN) for a one day scenario. Results are shown on a log-log scale. 


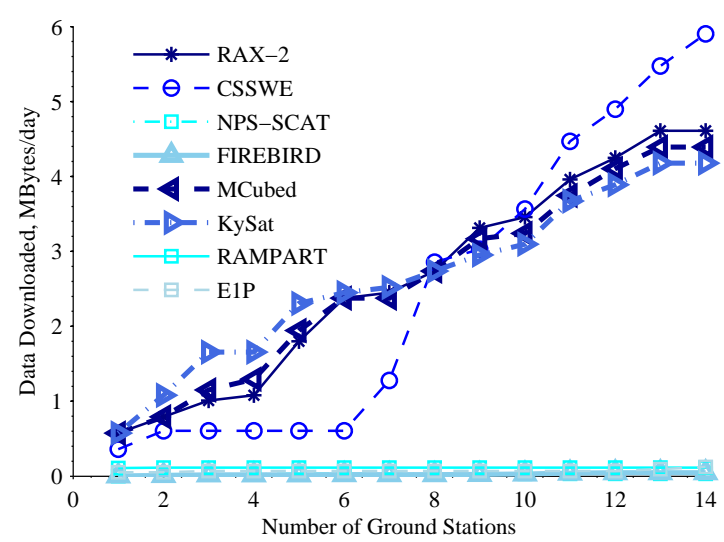

a) Low Data Rate $(<10$ kbits/sec) Missions

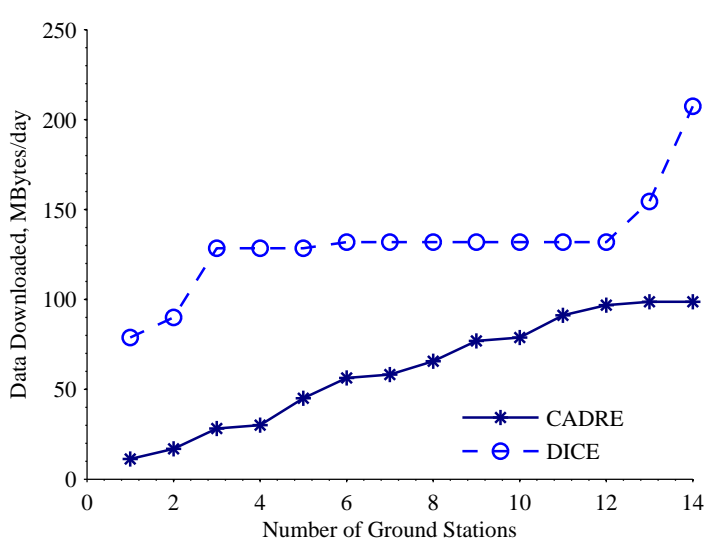

b) High Data Rate ( $>100$ kbits/sec) Missions

Figure 5. Sensitivity of data downloaded from optimized schedules to the number of ground stations in the LGN described in Section III.A.

smooth distributions, such as Ann Arbor, Michigan, and Wellington, New Zealand, while others are discontinuous, such as Sanford, North Carolina and Tokyo, Japan. The efficiency distributions highlight the great variability in efficiency characteristics of the ground stations and do not correspond to any sort of normal probability distribution. Thus, developing accurate models for the ground station efficiency is nearly impossible, however historical download efficiencies are available, which can be useful for operational planning.

We compare several strategies for modeling download efficiency in optimizing schedules in the presence of stochasticity by using historic data. The strategies are compared using the following two-step approach. First, schedules are optimized assuming a constant download efficiency is used for each station, selected according to the strategy, using the deterministic optimization formulation from Section II.B. The strategies include using the maximum efficiency ever realized for that station, the mean efficiency from historic data $(\eta=\mu)$, and perfect efficiency $(\eta=1)$. The first two strategies were selected as a simple approach to capturing the quality of the ground stations, such that higher-efficiency stations would be more likely to be selected for downloads than lower-efficiency stations. Once the optimal schedules are constructed, we perform a Monte Carlo analysis of the executed schedule, sampling download efficiencies randomly from the representative historic RAX-2 data. This enables us to gather statistical information about the download performance and robustness of the optimized download schedules for the strategies.

Figure 7 compares results of this stochastic analysis for the representative small satellite missions communicating to the LGN from Section III.A and the three strategies described above. For each strategy, we compare the minimum required download (which is independent of the strategy), expected download of the optimal solution with the given efficiency modeling strategy, and statistics on the mean and standard deviation (shown by the error bar) of executed schedules using realistic historic data. In general, the best strategy is the mean efficiency case $(\eta=\mu)$ because it results in the highest performance metric (data downloaded per day), although often the other two strategies perform similarly. Furthermore, the mean efficiency case tends to have smaller or equivalent standard deviations relative to the other strategies. These standard deviations range from 10-35\%, which provides bounds for the expected performance of the missions given realistic stochastic download efficiencies. These results motivate the need for improved techniques for modeling and solving scheduling problems when download efficiencies are stochastic.

\section{Conclusion and Future Work}

In this paper, we have developed an optimization formulation for the Single-Satellite Operational Scheduling Problem. This problem involves scheduling the operational tasks of a satellite to maximize the data collected and downloaded to a global ground station network. The formulation may be applied to problems with diverse satellite commodities (such as on-board energy and data and thermal states), subsystem functions (such as data collection by the payload and data downlink by the communication system), and mission constraints (such as satisfying buffer limitations and minimum data collection requirements). We have applied the optimization formulation to solve realistic small satellite mission scenarios and demonstrated the importance and advantages of considering the coupling between payload and download operations in satellite scheduling. By comparing the optimal results to the minimum download 


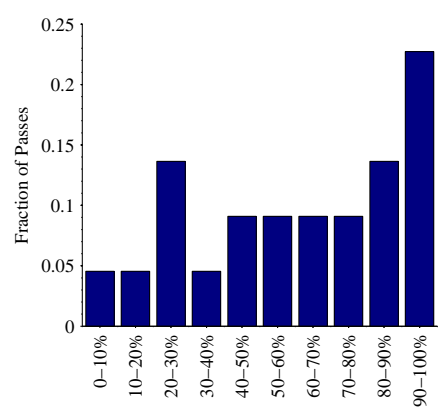

a) Adelaide, Australia

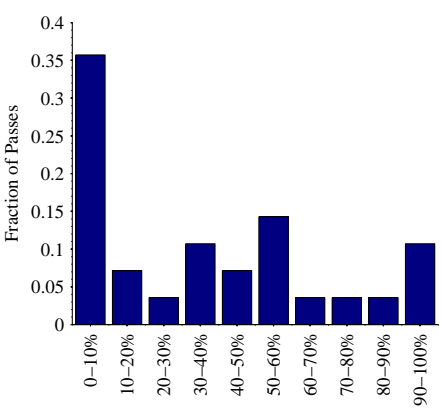

d) Sanford, North Carolina

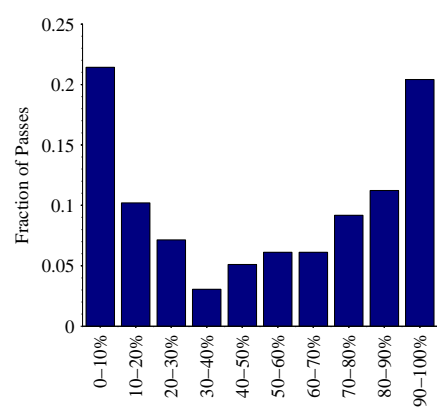

b) Ann Arbor, Michigan

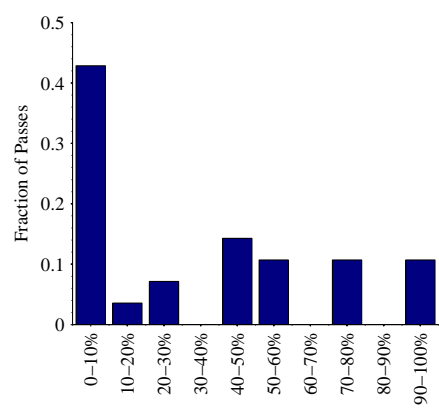

e) Tokyo, Japan

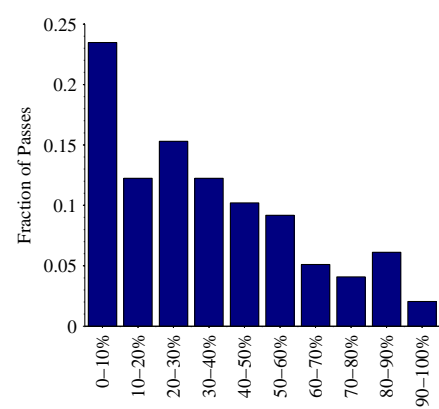

c) Fairbanks, Alaska

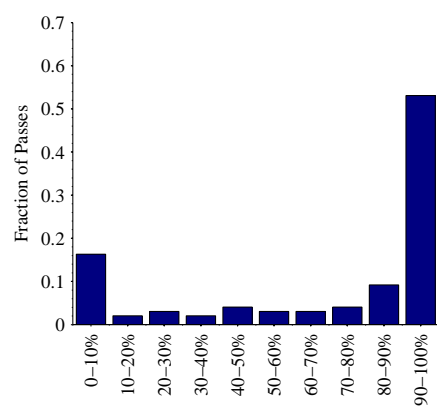

f) Wellington, New Zealand

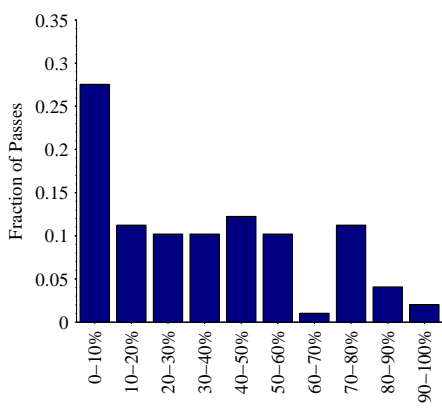

g) Menlo Park, California

Figure 6. Ground station efficiency distributions for RAX-2 mission. The efficiencies are computed for each satellite pass, the time where the satellite is continuously above the horizon.

requirements for each mission, we've demonstrated the advantages of optimizing operational schedules relative to using a conventional requirements-driven approach for operational planning.

Beyond optimizing existing satellite schedules, the optimization formulation presented in this paper enables satellite designers and operators to answer key operational and satellite design questions. For example, we've demonstrated how rapid computation of optimal solutions enables design space exploration that is useful to size batteries, and determine the impact of varying the size of the ground station network and data rates on the performance metrics. In addition, we've investigated the sensitivity of the optimized solutions to download efficiency stochasticity, a challenging characteristic of scheduling in the small satellite community. Monte Carlo experiments have enabled us to bound the expected performance for realistic mission scenarios using historic download efficiency data.

The foundational framework developed in this paper is extensible and modular to lay the ground work for several extensions. We plan to develop intelligent approaches to optimize space networks with multiple sources of stochasticity, such as energy availability, opportunistic payload collection, and download efficiencies. The problem of scheduling multiple independent or networked satellites communicating to shared ground networks subject to availability, priority, and cost constraints is an important issue to be addressed in future work. 


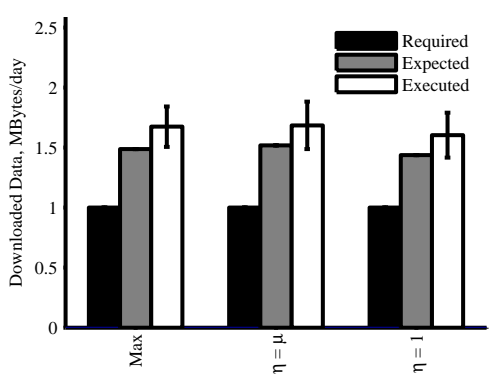

a) RAX-2

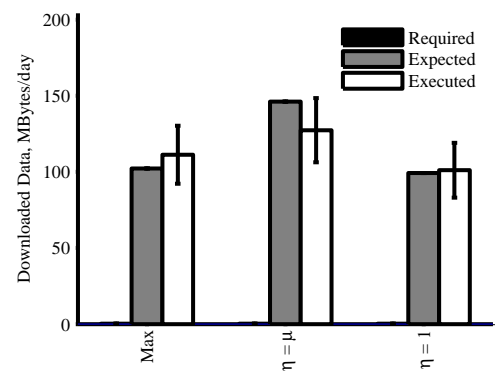

d) DICE

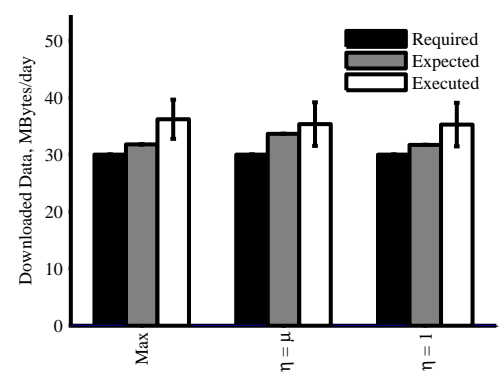

b) CADRE

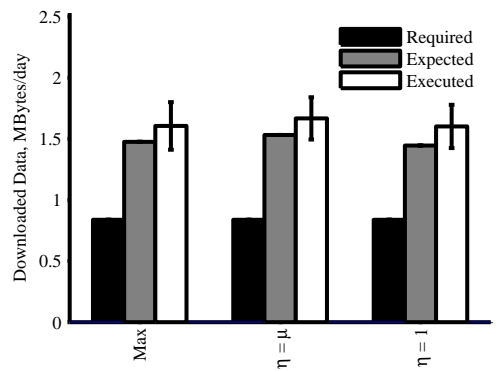

e) MCubed

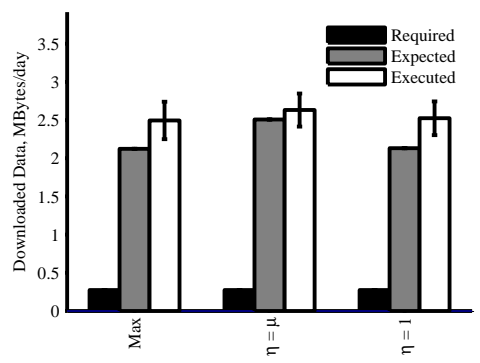

c) CSSWE

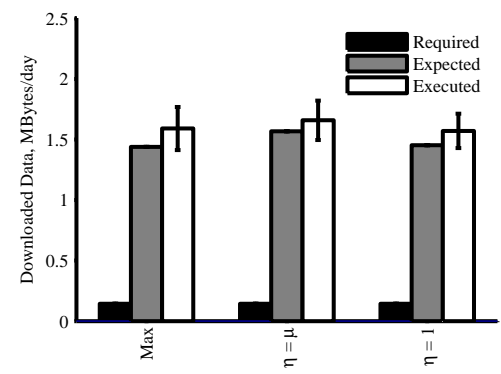

f) KySat

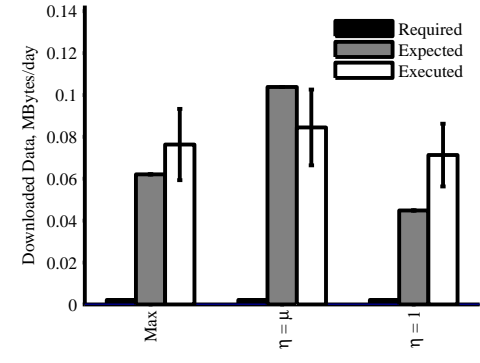

g) RAMPART

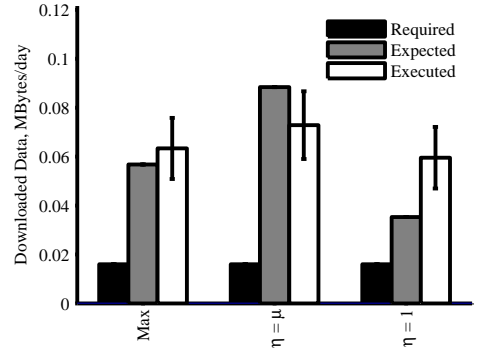

h) E1P

Figure 7. Comparison of strategies for modeling download efficiency (along the $\mathbf{x}$ axis) for representative missions from Table 1 downloading to the LGN from Section III.A for a two day planning horizon. For each strategy, we compare the minimum required download (which is independent of the strategy), expected download of the optimal solution with the given efficiency modeling strategy and statistics (mean and standard deviation, shown by the error bar) from a Monte Carlo analysis of executed schedules using realistic historic data (see Figure 6).

\section{Acknowledgements}

This research was sponsored by the National Science Foundation through NSF Award No. CNS-1035236 and the Canadian National Science and Engineering Research Council (NSERC). We would like to thank Dr. Amy Cohn and Kyle Gilson and the University of Michigan RAX Team for their input and support. We thank the CubeSat and amateur radio community for their support in completing the satellite and ground station surveys.

\section{References}

[1] Kirk Woellert, Pascale Ehrenfreund, Antonio J. Ricco, and Henry Hertzfeld. Cubesats: Cost-effective science and technology platforms for emerging and developing nations. Advances in Space Research, 47(4):663 - 684, 2011.

[2] Daniel N. Baker and S. Pete Worden. The large benefits of Small-Satellite missions. Transactions American Geophysical Union, 89(33):301+, August 2008.

[3] T. Moretto. Cubesat Mission to Investigate Ionospheric Irregularities. In Space Weather: The Journal of Research and 
Applications, November 2008.

[4] G. Earle and R. Davidson. Challenges and promise: CubeSat-based instrumentation for thermal plasma and neutral measurements. Fall AGU Meeting, December 2009.

[5] Therese Moretto and Robert M. Robinson. Small Satellites for Space Weather Research. Space Weather Journal, 6(5):S05007+, May 2008.

[6] D. M. Klumpar, H. E. Spence, B. A. Larsen, J. B. Blake, L. Springer, A. B. Crew, E. Mosleh, and K. W. Mashburn. FIREBIRD: A Dual Satellite Mission to Examine the Spatial and Energy Coherence Scales of Radiation Belt Electron Microbursts. American Geophysical Union (AGU) Fall Meeting, pages A8+, December 2009.

[7] James W. Cutler, John C. Springmann, Sara Spangelo, and Hasan Bahcivan. Initial flight assessment of the radio aurora explorer. In Proceedings of the 25th Annual Small Satellite Conference, Logan, Utah, August 2011.

[8] Garrett Skrobot. ElaNa educational launch of nanosatellite: Enhance education through space flight. In Proceedings of the 25th Annual Small Satellite Conference, August 2011.

[9] A. Ridley, J. Forbes, J. Cutler, A. Nicholas, J. Thayer, T. Fuller-Rowell, T. Matsuo, W. Bristow, M. Conde, D. Drob, L. Paxton, S. Chappie, M. Osborn, M. Dobbs, J. Roth, and Armada Mission Team. The Armada mission: Determining the dynamic and spatial response of the thermosphere/ionosphere system to energy inputs on global and regional scales. American Geophysical Union (AGU) Fall Meeting, pages A7+, December 2010.

[10] C. Swenson, J. C. Sojka, C. Fish, M. Larsen, B. Bingham, Q. Young, and S. Whitmore. The high-latitude dynamic e-field (hidef) explorer, a proposed network of 90 cubesats. QB-50 Workshop, Nov 2009.

[11] Martijn de Milliano and Chris Verhoeven. Towards the next generation of nanosatellite communication systems. Acta Astronautica, 66(9-10):1425 - 1433, 2010.

[12] J. Cutler and A. Fox. A framework for robust and flexible ground station networks. In Journal of Aerospace Computing, Information, and Communication, March 2006.

[13] Michel Vasquez and Jin-Kao Hao. A Logic-Constrained Knapsack Formulation and a Tabu Algorithm for the Daily Photograph Scheduling of an Earth Observation Satellite. Computational Optimization and Applications, 20:137-157, 2001. 10.1023/A:1011203002719.

[14] M. Lemaitre, G. Verfaillie, F. Jouhaud, J.-M. Lachiver, and N. Bataille. Selecting and scheduling observations of agile satellites. Aerospace Science and Technology, 6(5):367 - 81, Sept. 2002.

[15] William Wolfe and Stephen Sorensen. Three Scheduling Algorithms Applied to the Earth Observing Systems Domain. Management Science, 46(1):pp. 148-166, 2000.

[16] Baolin Sun, Lifei Mao, Wenxiang Wang, Xing Xie, and Qianqing Qin. Satellite mission scheduling algorithm based on Genetic Algorithm. volume 6795, page 67950U, 2007.

[17] William Martin. Satellite image collection optimization. Optical Engineering, 41(9):2083 - 2087, 2002.

[18] E. Bensana, M. Lemaitre, and G. Verfaillie. Earth observation satellite management. Constraints, 4:293-299, 1999. 10.1023/A:1026488509554.

[19] J. Pemberton and F. Galiber. A Constraint-Based Approach to Satellite Scheduling. pages 101 - 14, Providence, RI, 2001.

[20] Laura Barbulescu, Jean-Paul Watson, L. Whitley, and A. Howe. Scheduling space-ground communications for the Air Force satellite control network. Journal of Scheduling, 7:7-34, 2004.

[21] Fabrizio Marinelli, Salvatore Nocella, Fabrizio Rossi, and Stefano Smriglio. A Lagrangian Heuristic for Satellite Range Scheduling with Resource Constraints. In Dipartimento di Informatica, Universitita degli Studi di L'Aquila, Technical Report TRCS 004/2005, 2005.

[22] A. Globus and J. Crawford. A Comparison of Techniques for Scheduling Fleets of Earth-Observing Satellites. 2008.

[23] Kar-Ming Cheung, C.H. Lee, W.B. Gearhart, T. Vo, and S. Sindi. Link-capability driven network planning and operation. volume 7, page 3281, Piscataway, NJ, USA, 2002.

[24] Sara Spangelo and James Cutler. Analytic modeling framework and simulation toolkit for space network communication capacity assessment. IEEE Transactions on Aerospace and Electronic Systems (In Progress), 2011.

[25] Sara Spangelo, James Cutler, Kyle Gilson, and Amy Cohn. Optimization-based scheduling for the single-satellite, multiground station communication problem. Operations Research (In Progress), 2011.

[26] IBM. IBM ILOG CPLEX Optimization Studio. http://www-01.ibm.com/software/integration/optimization/cplexoptimization-studio/, March 2010.

[27] Small Satellite Survey. http://gs.engin.umich.edu/sat_survey, 2011.

[28] CubeSat Ground Station Survey. http://gs.engin.umich.edu/gs_survey, 2009-2011.

[29] James Cutler, Peder Linder, and Armando Fox. A federated ground station network. In Proceedings of SpaceOps 2002, Houston, TX, October 2002. AIAA.

[30] Genso. http://www.amsat.org/amsat-new/satellites/history.php, July 2012. 